octreotide and docetaxel. After a year, nivolumab was added. We measured the levels of nivolumab in her pleural fluid and serum to determine whether or not there was response and penetration. Outcomes were analyzed based on correlations of PD-L1 expression, clinicopathologic parameters, and CT scans, performed every six weeks. PD-1 negative carcinoid tumors have a certain response to PD-1 inhibitors. As planned in our hypothesis, this can be related to either an immune reaction in carcinoid type tumors, or possible alternative pathways of response. The complete mechanism of action remains unknown. This warrants further studies.

\section{SYSTEMS BIOLOGY OF HUMAN AGING - NETWORK MODEL 2018}

\section{J. Furber, Legendary Pharmaceuticals}

This network schema is presented to aid in conceptualizing the many processes of aging, the causal chains of events, and the interactions among them, including feedback (vicious cycles). Contemplation of this network suggests promising intervention points for therapy development. This diagram is maintained on the Web as a reference for researchers and students. Content is updated as new information comes to light. www.LegendaryPharma.com/chartbg.html At first glance the network looks like a complicated web. However, as a conceptual summary, in one view, we can see how the many biogerontological processes relate to each other. Importantly, examination of these relationships allows us to pick out reasonably plausible causal chains of events. Within these chains, we can see age-related changes or accumulations that appear to be promising targets for future therapy development. Especially harmful is damage to the body's regeneration and repair systems, because they normally repair damage to other structures and systems. The many observable signs of human senescence have been hypothesized by various researchers to result from several primary causes. Inspection of the biochemical and physiological pathways associated with age-related changes and with the hypothesized causes reveals several parallel cascades of events that involve several important interactions and feedback loops. This network model includes both intracellular and extracellular processes. It ranges in scale from the molecular to the whole-body level. Effects due to externalities, lifestyle, environment, and proposed interventions are highlighted around the margins of the network.

\section{TELOMERE LENGTH GENETIC VARIATION AND AGING: A NATURAL EXPERIMENT IN 250,000 OLDER PEOPLE}

C. Kuo ${ }^{1}$, L. Pilling ${ }^{2}$, G. Kuchel ${ }^{3}$, L. Ferrucci ${ }^{4}$, 1. University of Connecticut Health, 2. University of Exeter Medical School,

3. University of Connecticut, 4. National Institute on Aging

Telomere length is a core factor in aging biology and interventions to lengthen telomeres have been advocated for anti-aging. We aimed to test whether genetically determined variation in telomere length is associated with a wide range of aging-related conditions. Studying inherited genetic variants for telomere length minimizes confounding from lifestyle factors, and excludes feedback effects due to developing disease (i.e. excludes reverse causation). Subjects are naturally randomized into subgroups of these variants, which creates a natural randomized trial (a 'Mendelian randomization' trial) and provides more robust evidence than conventional observational studies. Associations between genetic variants strongly associated with telomere length (in TERC, TERT, NAF1, OBFC1 and RTEL1) and aging-related outcomes were tested in 250000 participants of European descent aged 60 and older at measurement. The odds ratio per standard deviation change in telomere length (approx. 4,000 base pairs) was $\mathrm{OR}=1.23$ (95\% CI: $1.10-1.37)$ for developing any cancer. Genetically longer telomeres were also associated with hypertension (OR=1.18, 95\% CI: 1.09-1.29). There was suggestive evidence of increased participant mortality with genetically longer telomeres (HR $=1.18$, 95\% CI: $1.03-$ 1.35). However, no associations were found with healthy aging outcomes including falls, fractures, depression, frailty, sarcopenia, and hospitalization for pneumonia or delirium. We conclude that genetically increased telomere length is associated with a higher risk of cancers but no improvement in measures of healthy aging.

\section{TESTING THE LIMIT TO HUMAN LIFESPAN HYPOTHESIS WITH DATA ON SUPERCENTENARIANS}

N. Gavrilova, L. Gavrilov, NORC at the University of Chicago

The topic of limit to human lifespan received a lot of attention after publication in Nature suggesting its existence and recent publication in Science with the opposite conclusion. In this study, data on supercentenarians (aged $110+)$ are used to test two expected outcomes in case the limit does exist: (1) lack of growth in longevity records for subsequent birth cohorts and (2) accelerated growth of mortality at extreme old ages. Data are taken from two sources: International Database on Longevity (IDL) and Gerontology Research Group (GRG) database. We found that for extinct birth cohorts, maximum reported age at death is increasing in subsequent cohorts but with slower and statistically insignificant pace for cohorts born after 1879 than for earlierborn cohorts. Comparison of models describing mortality trajectories after age 110 years using the Akaike information criterion found that mortality of earlier birth cohorts (born before 1885) were best fitted by exponential model (flat mortality) while for later birth cohorts (born in 1885-1898), the Gompertz model showed better fit. These results demonstrate that hazard rates after age 110 years do not stay constant for cohorts born after 1885 . It turns out that supercentenarians born after 1885 show steeper mortality growth with age compared to ages below 110 years, which suggests possible mortality acceleration. Slow progress in maximum reported age at death and mortality acceleration at extreme old ages for later birth cohorts may indicate that possibility of temporary limit to human lifespan cannot be completely excluded. Supported by NIA grant R21AG054849.

\section{THE CLOSE INVERSE RELATIONSHIP BETWEEN MALE AND FEMALE HEIGHT AND LIFE EXPECTANCY}

J. Lillis ${ }^{1}$, S. Marson ${ }^{2}$, T. Samaras ${ }^{3}$, 1. University of North Carolina at Pembroke, 2. UNCP, 3. Reventrophy Associates

Many papers have provided reasons for why women live longer than men. These include XX vs. XY chromosomes, sex hormones, and better care of their health. In addition, males experience more deaths from homicides and risky 